\title{
Extrathyroid carcinoma showing thymus-like differentiation (CASTLE): a new case report and review of the therapeutic role of neck dissection and radiotherapy
}

Kyu Young Choi ${ }^{1}$, Mi Jung Kwon ${ }^{2}$, Hye Kyung Ahn ${ }^{3}$, Jin Hwan Kim and Dong Jin Lee ${ }^{1 *}$

\begin{abstract}
We present here a case of extrathyroid CASTLE (the third case reported in the English literature) treated with excision and neck dissection without radiotherapy. Also, we reviewed the literature and analyzed the therapeutic results of each treatment modality for CASTLE. A 27-year-old male had initially presented with a painless, right neck mass for 2 months. Computed tomography of the neck showed a $3.8 \times 3.2 \times 3.8 \mathrm{~cm}$ heterogeneously enhancing mass at right level lla, and no definite thyroid lesion was found. An excisional biopsy was done and the pathologic diagnosis was CASTLE. Then we performed a right modified radical neck dissection and right thyroid lobectomy. After three years, no evidence of tumor recurrence was noted. Total excision followed by neck dissection could be a sufficient surgical treatment option for CASTLE. Postoperative radiotherapy might be an alternative treatment option for neck dissection in patients with positive nodal status.
\end{abstract}

Keywords: Carcinoma showing thymus-like differentiation, CASTLE, thyroid gland, neck dissection, radiotherapy

\section{Background}

Carcinoma showing thymus-like differentiation (CASTLE) is a rare tumor of the thyroid gland or adjacent soft tissues of the neck [1]. First described as an intrathyroid epithelial thymoma by Miyauchi et al. in 1985 [2], these lesions were later designated CASTLE by Chan and Rosai, who proposed an origin from ectopic thymus, or from thymopharyngeal duct or branchial pouch remnants in the thyroid gland and neck [3]. The majority of tumors occur in the thyroid gland, usually in the lower portion, and they rarely arise in the extrathyroidal soft tissue of the neck. The histopathology of CASTLE is characterized by an expansive growth pattern, thick fibrous bands dividing the tumor nests, the presence of lymphocytes and rare or infrequent mitoses [4]. Generally, the treatment is surgical excision with or without radiotherapy, but the definitive

\footnotetext{
* Correspondence: djlee@hallym.or.kr

'Department of Otorhinolaryngology-Head and Neck Surgery, Hallym University College of Medicine, Kangnam Sacred Heart Hospital, Daerim-1dong, Yeongdeungpo-gu, Seoul 150-950, South Korea

Full list of author information is available at the end of the article
}

treatment remains uncertain, due to the rarity of the disease.

According to reports in the English-language literature, less than 30 cases of CASTLE tumors have been reported, and only two cases were extrathyroid tumors [5]. One case was reported by Ahuja in 1998, in which a female patient with a lateral neck CASTLE was treated by resection and radiotherapy without recurrence [6], and the other case was described by Luo, in which a lateral neck CASTLE was treated without locoregional recurrence by surgical excision and radiotherapy [5].

Here, we report a case of extrathyroid CASTLE, which was diagnosed by excisional biopsy, successfully treated by unilateral neck dissection with ipsilateral thyroid lobectomy, without concomitant radiotherapy.

\section{Case presentation}

A 27-year-old male was admitted to our department with a palpable neck mass that appeared two months ago. He denied any other symptoms. His past medical history was significant only for left chronic otitis media with mild hearing disturbance, and both chronic sinusitis, which was 
treated surgically by endoscopic sinus surgery seven years ago. On physical examination, the 3-cm-sized mass was hard and fixed, locating at right level IIa. No obvious lesion was found in the oral cavity, nasopharynx, oropharynx or hypopharynx. Laboratory tests showed a white blood cell count of $7,480 \mathrm{~mm}^{3}$ with $64.2 \%$ neutrophils, and the serum concentrations of thyroxine, thyroidstimulating hormone and thyroglobulin were all normal. Neck ultrasonography revealed multiple tiny cystichypoechoic nodules in both thyroid glands, and a $3.8 \times$ $3.2 \times 3.8 \mathrm{~cm}$ mass with heterogeneous echogenicity in the right submandibular area. Computed tomography (CT) with enhancement of the neck showed the same-sized heterogeneously enhancing mass at right level IIa (Figure 1). Ultrasonogram guided fine-needle aspiration (FNA) biopsy of the mass was performed and only atypical plump spindle cells were found. We performed a second FNA biopsy after two weeks. The cytologic examination showed plump epitheloid and spindle cells with infiltration of lymphoid cells and eosinophils. An immunohistochemical stain of the cytology showed that the tumor was positive for keratin, high-molecular keratin, vimentin and galectin3 , and focally positive for Factor VIII.

To make a definite diagnosis, we scheduled an excisional biopsy of the mass. Under general anesthesia, a skin incision over the mass and subsequent dissection of the platysma muscle was done, and the tumor appeared just inferior to the right submandibular gland. The tumor was well encapsulated with several lymph nodes around it. Excision of the mass along with six right level IIa lymph nodes was performed. No other specific intraoperative

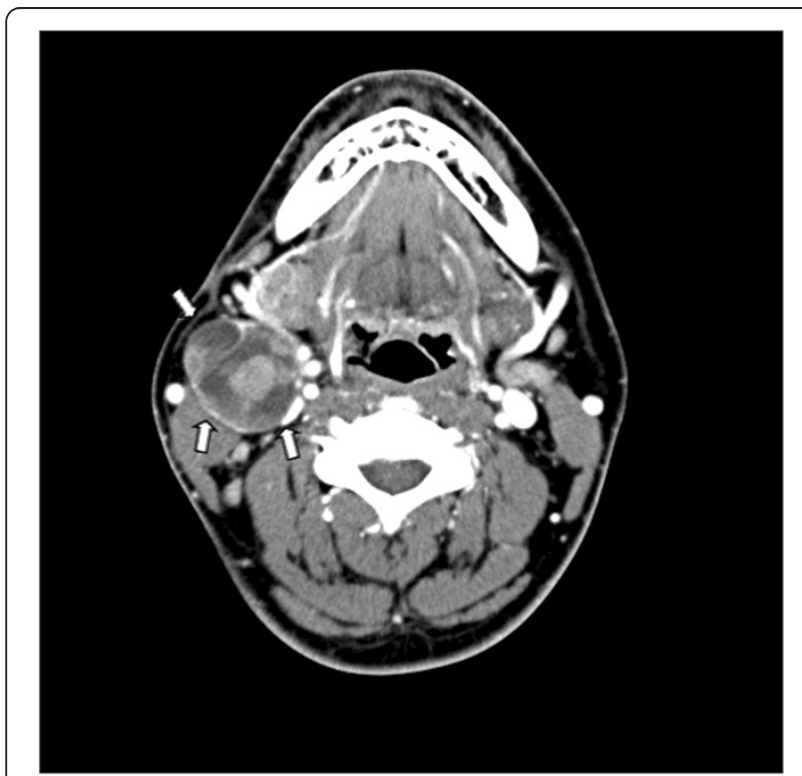

Figure 1 Computed tomography (CT) findings. $C T$ with enhancement showing heterogeneously enhancing mass at right level II (arrows, tumor margin). findings were noticed and the frozen biopsy revealed not much and no malignant tissue was found. One week after, a final report of the permanent pathology was made and the diagnosis was carcinoma showing a thymus-like element. Histological examination of the mass showed an infiltrative neoplasm composed of epithelial cell nests separated by thick fibrous septa, with an accompanying infiltrate of small lymphocytes and eosinophils (Figure 2). Immunohistochemical stains of the mass for CD5, cytokeratin and P63 were positive, and negative for thyroid transcription factor-1. The six lymph nodes had no tumor present.

The patient refused radiotherapy, but agreed on complete surgical eradication of the disease. Because of the patient's young age, we scheduled a right modified radical neck dissection, along with ipsilateral thyroid lobectomy to rule out a thyroid origin. Preoperative positron emission tomography-computed tomography (PET-CT) showed no metabolic evidence of malignancy, and an ultrasonogram guided thyroid nodule FNA biopsy result was non diagnostic. Finally, two months after the first operation, a right modified radical neck dissection (levels I to V) and ipsilateral thyroid lobectomy were performed. A thyroid lobectomy was added to our surgery because most CASTLE tumors are intrathyroid, and also to give a definite diagnosis of the patient's thyroid nodule. On intraoperative inspection and palpation of the thyroid, no noticeable intrathyroid mass was found. The surgery was finished without difficulty, and no other specific intraoperative findings were noted. The pathologic report of the right thyroid lobe was multinodular goiter and no definite neoplastic lesion was identified. All the lymph nodes in right levels I to $\mathrm{V}$ were non-neoplastic (reactive hyperplasia). The patient was discharged on the ninth hospital day without any postoperative complications, and was then regularly followed up in the outpatient department. After six months, a follow up PET-CT was taken and no metabolic evidence of regional tumor recurrence or metastasis was reported. At the most recent follow-up, i.e. three years after the second operation, no evidence of tumor recurrence was present.

\section{Discussion}

CASTLE is a rare malignant neoplasm that histologically resembles thymic carcinoma, and arises in the thyroid gland or adjacent soft tissue of the neck. During embryological development, primordial thymic tissue derived from the third and fourth branchial pouches migrates through the neck and into the mediastinum [7]. Persistence of thymic remnants along this path can manifest as ectopic thymic tissue [3]. CASTLE is postulated to arise from either this ectopic tissue, from remnants of the thymopharyngeal duct or from ultimobranchial body remnants within the thyroid gland $[3,8]$. According to previous 


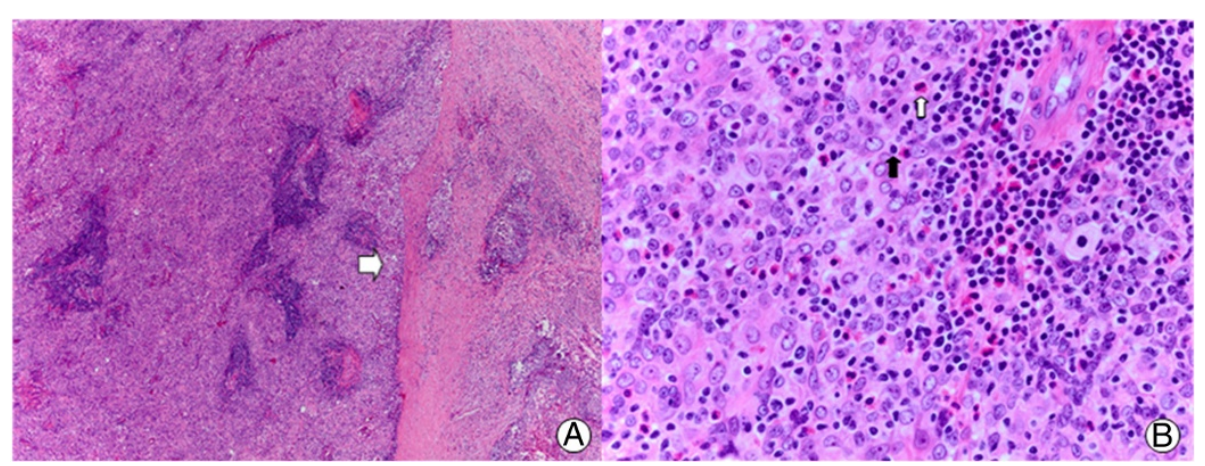

Figure 2 Histopathological features of the tumor. (A) Tumor cell nests separated by fibrous bands (white arrow, hematoxylin and eosin stain; original magnification $\times 40$ ), (B) with accompanying lymphocyte (black arrow) and eosinophil (white arrow) infiltration (hematoxylin and eosin stain; original magnification $\times 400$ ).

reports, most CASTLE tumors are located in the thyroid gland, especially the lower portion. From a review of literature, only three CASTLE tumors have been reported in the lateral neck including this case [5,6]. Table 1 summarizes the clinical characteristics, treatments and clinical outcomes of the three patients with extrathyroidal CASTLE.

An FNA biopsy seems to offer limited diagnostic value for CASTLE. Youens et al. identified 27 cases of reported CASTLE that mentioned an FNA biopsy [1]. Reported cytological differential diagnoses vary, with the majority of cases being identified as a malignant tumor, without further definite classification. One reported case was identified as suggestive of spindle cell neoplasm, one was identified as a papillary carcinoma, and one was correctly identified as CASTLE preoperatively. In 23 of 27 reported cases, CASTLE was correctly identified as malignant, and in one case, the FNA was negative. In our case, the FNA biopsy showed epitheloid and spindle cells with infiltration of lymphoid cells and eosinophils. CASTLE can be definitively diagnosed only by experienced pathologists through histologic examination. CASTLE produces a lobulated and expansive growth pattern with fibrous septa dividing the tumor, large vesicular nuclei with prominent nucleoli, a low mitotic count, and may be associated with lymphoplasmacytic infiltration $[2,3,9]$, as seen in our patient (Figure 2). Small focal squamous differentiation in whorled clusters, resembling Hassall's corpuscles, may also be seen [5].

In their study, Luo et al. found 26 reported cases of CASTLE, 25 intrathyroid cases, one extrathyroid case, and added one extrathyroid CASTLE, which they reported [5]. The 25 cases of intrathyroid CASTLE were treated with surgical excision (thyroidectomy, neck dissection, etc.) with or without radiotherapy. A review of all reported cases showed that 16 of 27 patients had no evidence of recurrence at follow-up after treatment, and 10 of 27 had a recurrence. There was one case of recurrence with initial misdiagnosis. Four of the ten recurrent cases showed no evidence of recurrence after salvage treatment; one patient

Table 1 Clinical characteristics, treatments, and outcomes of patients with extrathyroidal origin CASTLE

\begin{tabular}{|c|c|c|c|}
\hline Cases & Ahuja et al. [6] & Luo et al. [5] & This case \\
\hline Age (years)/sex & 67/female & 47/male & 27/male \\
\hline Tumor size & $9 \times 7 \times 5 \mathrm{~cm}$ & $5 \times 5 \times 4 \mathrm{~cm}$ & $3.8 \times 3.8 \times 3.2 \mathrm{~cm}$ \\
\hline Location in neck & Left & Left & Right \\
\hline Cystic changes & Not described & Partial & Partial \\
\hline Inflammatory cell infiltration & Numerous lymphocytes & Lymphocytes & Abundant lymphocytes and eosinophils \\
\hline Adjacent LNs & $\begin{array}{l}\text { Multiple enlarged LNs adjacent } \\
\text { to the mass }\end{array}$ & $\begin{array}{l}\text { Several small LNs surrounding } \\
\text { the mass }\end{array}$ & Multiple enlarged LNs adjacent to the mass \\
\hline LN pathology & LN metastasis $(1 / 22)$ & No tumor & No tumor \\
\hline Treatment & $\begin{array}{l}\text { Mass excision, LN excision, } \\
\text { radiotherapy }\end{array}$ & $\begin{array}{l}\text { Mass excision, LN excision, } \\
\text { radiotherapy }\end{array}$ & $\begin{array}{l}\text { Mass excision, right modified radical neck } \\
\text { dissection (levels I-V) }\end{array}$ \\
\hline Outcome & No recurrence & No recurrence & No recurrence over 3-year period \\
\hline
\end{tabular}


died of a cerebrovascular disorder, and five died of the disease with local recurrence or distant metastasis to the lung or liver. Most of the recurrences were noted in patients with invasion of the neck lymph nodes or surrounding tissue. Two cases of extrathyroid CASTLE were treated by resection and radiotherapy, and neither showed locoregional recurrence.

There is no consensus on the management of CASTLE, due to the rarity of the disease, but complete surgical excision with or without radiotherapy seems necessary to improve the long-term survival rate and reduce the locoregional recurrence rate. Table 2 summarizes the treatment modalities and therapeutic results of CASTLE, from a review of the literature. We added six new cases from the recent literature for which we could find the treatment modalities and therapeutic results [10-13]. In cases with a tumor excision only, tumor recurrence occurred in six cases and there was no evidence of disease (NED) for seven cases. But for cases who had either radiation therapy or neck dissection after complete excision, recurrence occurred only for three cases and there was NED for nine cases. On the basis of these case reviews, we noticed that excision with either radiation or neck dissection showed better results than excision only. Additionally, if there is a positive node in the neck dissection specimen, postoperative radiation might be helpful as this adjuvant therapy reduced the recurrence rate from $100 \%$ to $57 \%(1 / 1$ to $4 / 7)$. In contrast, if there is no metastatic node in the neck dissection specimen, postoperative radiation therapy does not seem necessary as recurrence has never occurred in node negative cases after neck dissection.

In summary, the most successful treatment from a review of the literature was complete surgical excision followed by neck dissection with or without postoperative adjuvant radiation therapy depending on nodal state. In

Table 2 Treatment modalities and therapeutic results of reported CASTLEs [5,6,10-13]

\begin{tabular}{lcccc}
\hline Treatment modality & & Recurrence & NED \\
\hline Excision only & & 6 & & 7 \\
Excision + RT & & 2 & & 4 \\
Excision + RT* & 0 & & 1 \\
Excision + ND & $\mathrm{N} \mathrm{(+)}$ & 1 & $\mathrm{~N}(+)$ & 0 \\
& $\mathrm{~N}(-)$ & 0 & $\mathrm{~N}(-)$ & 3 \\
Excision + ND* & $\mathrm{N} \mathrm{(-)}$ & 0 & $\mathrm{~N} \mathrm{(-)}$ & 1 \\
Excision + ND + RT & $\mathrm{N}(+)$ & 4 & $\mathrm{~N} \mathrm{(+)}$ & 2 \\
& $\mathrm{~N} \mathrm{(-)}$ & 0 & $\mathrm{~N} \mathrm{(-)}$ & 2 \\
Excision + ND + RT & $\mathrm{N}(+)$ & 0 & $\mathrm{~N} \mathrm{(+)}$ & 1 \\
Total & & 13 & & 21
\end{tabular}

*The three cases of extra-thyroid CASTLE.

$\mathrm{N}(+)$, node positive in neck dissection specimen; $\mathrm{N}(-)$, node negative in neck dissection specimen; ND, neck dissection; NED, no evidence of disease; $\mathrm{RT}$, radiation therapy. our case, complete excision by neck dissection without radiotherapy achieved a positive result as there was no metastatic node in the neck specimen. Interestingly, all extrathyroid CASTLE cases ("' in Table 2) showed NED. All three cases were treated by excision with either radiotherapy or neck dissection. The reason for the better prognosis compared with intrathyroid CASTLE might be the isolated origin site of extrathyroid CASTLE, and the higher feasibility of complete excision than intrathyroid CASTLE, which extends to the mediastinum. Extrathyroid CASTLE is difficult to diagnose, and there is no reported suitable treatment. In this case, the patient was diagnosed by excisional biopsy, and a subsequent unilateral neck dissection with ipsilateral thyroid lobectomy achieved a disease-free state. However, a longer follow-up in a larger series of patients is needed to support our result.

\section{Conclusions}

We present a case of extrathyroid CASTLE, which was successfully treated by excision plus unilateral neck dissection. From a review of the literature, the most successful treatment of CASTLE is complete surgical excision followed by neck dissection with or without postoperative adjuvant radiation therapy depending on nodal state.

\section{Consent}

Written informed consent was obtained from the patient for publication of this case and for the accompanying images.

\section{Abbreviations}

CASTLE: carcinoma showing thymus-like differentiation; $\mathrm{CT}$ : computed tomography; FNA: fine-needle aspiration; NED: no evidence of disease; PET-CT: positron emission tomography-computed tomography.

\section{Competing interests}

None of the authors involved in manuscript preparation have any competing interests regarding the manuscript itself, nor are there any financial or moral conflicts. None of the authors received support in the form of grants, equipment or pharmaceutical items.

\section{Authors' contributions}

KYC collected data and drafted the manuscript. MJK and HKA supervised the pathology and managed the figures. JHK supervised the writing of the manuscript. DJL conceived of the study and corrected and revised the manuscript. All authors read and approved the final manuscript.

\section{Author details}

${ }^{1}$ Department of Otorhinolaryngology-Head and Neck Surgery, Hallym University College of Medicine, Kangnam Sacred Heart Hospital, Daerim-1dong,

Yeongdeungpo-gu, Seoul 150-950, South Korea. 'Department of Pathology, Hallym University College of Medicine, Hallym University Sungsim Hospital,

Pyeongan-dong, Dongan-gu, Anyang-si, Gyeonggi-do 431-796, South Korea. ${ }^{3}$ Department of Pathology, Hallym University College of Medicine,

Kangnam Sacred Heart Hospital, Daerim-1dong, Yeongdeungpo-gu, Seoul 150-950, South Korea.

Received: 9 May 2014 Accepted: 20 July 2014

Published: 3 August 2014 


\section{References}

1. Youens KE, Bean SM, Dodd LG, Jones CK: Thyroid carcinoma showing thymus-like differentiation (CASTLE): case report with cytomorphology and review of the literature. Diagn Cytopathol 2011, 39:204-209.

2. Miyauchi A, Kuma K, Matsuzuka F, Matsubayashi S, Kobayashi A, Tamai H, Katayama S: Intrathyroidal epithelial thymoma: an entity distinct from squamous cell carcinoma of the thyroid. World J Surg 1985, 1:128-135.

3. Chan JK, Rosai J: Tumors of the neck showing thymic or related branchial pouch differentiation: a unifying concept. Hum Pathol 1991, 4:349-367.

4. Cheuk W, Jacobson AA, Chan JKC: Spindle epithelial tumor with thymus-like element: a distinctive malignant thyroid tumor with significant metastatic potential. Mod Pathol 2000, 13:1150-1155.

5. Luo CM, Hsueh C, Chen TM: Extrathyroid carcinoma showing thymus-like differentiation (CASTLE) tumor - a new case report and review of literature. Head Neck 2005, 27:927-933.

6. Ahuja AT, Chan ESY, Allen PW, Lau KY, King W, Metreweli C: Carcinoma showing thymic-like differentiation (CASTLE tumor). Am J Neuroradiol 1998, 19:1225-1228.

7. Yamazaki M, Fujii S, Daiko H, Hayashi R, Ochiai A: Carcinoma showing thymus-like differentiation (CASTLE) with neuroendocrine differentiation. Pathol Int 2008, 58:775-779.

8. Reimann JD, Dorfman DM, Nose V: Carcinoma showing thymus like differentiation of the thyroid (CASTLE): a comparative study: evidence of thymic differentiation and solid cell nest origin. Am J Surg Pathol 2006 8:994-1001.

9. Mizukami $Y$, Kurumaya $H$, Yamada $T$, Minato $H$, Nonomura A, Noguchi $M$ Matsubara F: Thymic carcinoma involving the thyroid gland: report of two cases. Hum Pathol 1995, 26:576-579.

10. Alifano M, Boudaya MS, Dinu C, Kadiri H, Regnard JF: Carcinoma showing thymus-like elements invading the trachea. J Thorac Cardiovasc Surg 2006, 132:191-192.

11. Cappelli C, Tironi A, Marchetti GP, Pirola I, De Martino E, Delbarba A, Castellano M, Rosei EA: Aggressive thyroid carcinoma showing thymic-like differentiation (CASTLE): case report and review of the literature. Endocr J 2008, 55:685-690.

12. Sun $T$, Wang $Z$, Wang J, Wu Y, Li D, Ying $H$ : Outcome of radical resection and postoperative radiotherapy for thyroid carcinoma showing thymus-like differentiation. World J Surg 2011, 35:1840-1846.

13. Veits L, Mechtersheimer G, Steger C, Freitag J, Mikuz G, Schmid KW, Hofmann W, Schirmacher P, Hartmann A, Rieker RJ: Chromosomal imbalances in carcinoma showing thymus-like elements (CASTLE). Virchows Arch 2011, 459:221-226.

doi:10.1186/1477-7819-12-247

Cite this article as: Choi et al.: Extrathyroid carcinoma showing thymus-like differentiation (CASTLE): a new case report and review of the therapeutic role of neck dissection and radiotherapy. World Journal of Surgical Oncology 2014 12:247.

\section{Submit your next manuscript to BioMed Central and take full advantage of:}

- Convenient online submission

- Thorough peer review

- No space constraints or color figure charges

- Immediate publication on acceptance

- Inclusion in PubMed, CAS, Scopus and Google Scholar

- Research which is freely available for redistribution 